\title{
Introduzione ai sistemi di geoscambio
}

\author{
Matteo Cultrera \\ Transmark Renewables - NL \\ m.cultrera@transmark-renewables.com
}

Nei numeri precedenti di Acque Sotterranee sono stati introdotti i Sistemi Geotermici ed e' stata suggerita una definizione che tenesse conto dell'entalpia (temperatura), della conducibilità idraulica e del tipo di trasporto di calore (conduttivo Vs advettivo) (Cultrera, 2013).

In questo numero si introducono i sistemi di geoscambio, ovvero quei sistemi geotermici attraverso i quali non si produce energia elettrica mediante lo sfruttamento diretto del calore, bensì si sfruttano le temperature costanti del sottosuolo per la climatizzazione dei diversi ambienti (Huenges, 2010) (Fig. 1).

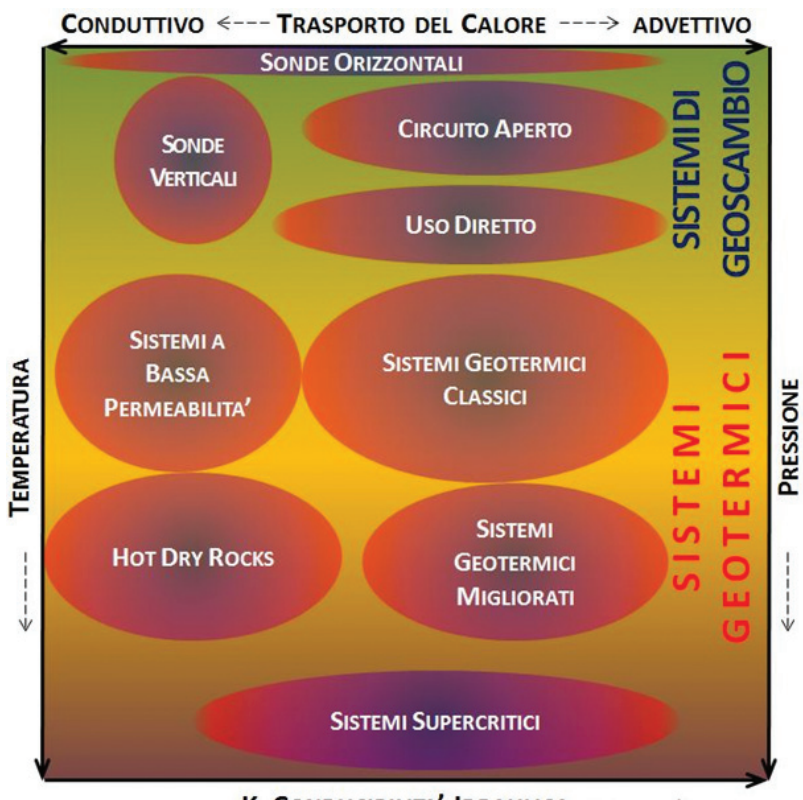

K, CONDUCIBILITA' IDRAULICA -------

Fig.1: classificazione dei sistemi geotermici per i diversi utilizzi delle risorse geotermiche (Cultrera, 2013)

Lo sfruttamento dei sistemi di geoscambio è reso possibile mediante il ricorso a 3 gruppi di tecnologie, all'interno di ognuna delle quali sono possibili eventuali ulteriori suddivisioni:

1) Sistemi a circuito chiuso (closed loop):

1a) Sonde verticali (Borehole Heat Exchanger);

1b) Sonde orizzontali (Horizontal Closed Loop);

1c) Sonde radiali (Radial Closed Loop)

2) Sistemi a circuito aperto (Open Loop)

3) Scambio diretto;

I sistemi di geoscambio sfruttano le temperature costanti nell'arco dell'anno presenti nel sottosuolo; infatti, le oscillazioni di temperatura giornaliere possono essere considerate trascurabili già al di sotto del primo metro di profondità, mentre oltre i 6-8 metri le oscillazioni annuali diventano poco significative (Fig. 2); il range di profondità qui descritto dipende principalmente - ma non solo - dalla diffusività termica del terreno, dalla presenza di acque sotterranee, dalla temperatura atmosferica al suolo. Oltre i 15-20 m di profondità le temperature nel sottosuolo sono condizionate dal gradiente geotermico locale (gradiente normale pari a $3^{\circ} \mathrm{C} / 100$ m) (Banks, 2008)

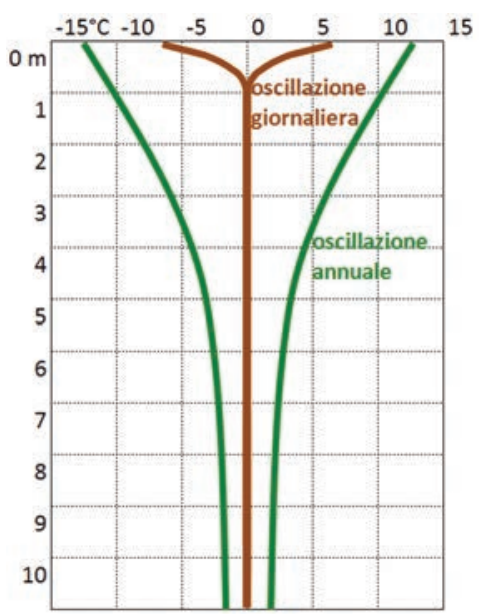

Fig.2: esempio di variazione delle temperature giornaliera e annuale nel sottosuolo.

In Italia ed in condizioni di gradiente geotermico normale, i sistemi geotermici sfruttati per il geoscambio beneficiano di temperature generalmente comprese mediamente tra $10-12{ }^{\circ} \mathrm{C}$ ed i $18-20{ }^{\circ} \mathrm{C}$, per profondità non superiori ai 100 $\mathrm{m}$. Tali temperature consentono di estrarre facilmente quelle calorie/frigorie necessarie per la climatizzazione degli ambienti. Il gap termico per la climatizzazione è generalmente contenuto e il conseguente lavoro necessario per il trasferimento del calore è vantaggioso in termini di dispendio di energia elettrica (Freeston, 1985).

Il lavoro necessario per operare il trasferimento del calore avviene mediante l'accoppiamento ad una pompa di calore. In tal caso si parla di «pompa di calore geotermica» (Ground Source Heat Pump, GSHP). Questa consente di trasferire l'energia termica da una sorgente a temperatura più bassa ad una sorgente a temperatura più alta. Ovviamente anche il percorso inverso (da temperatura alta a temperatura bassa) è sempre possibile (Huttrer, 1997). 
Per valutare la prestazione di una pompa di calore si ricorre al «coefficiente di prestazione» (Coefficient Of Performance, COP). Il COP di una pompa di calore viene valutato mediante il confronto tra la potenza termica utile in uscita dal condensatore $(\mathrm{Q})$ con la potenza fornita al compressore (W) (Egg, 2011).

In condizioni di riscaldamento il COP e> definito dall'equazione [1]:

$$
C O P_{\text {heating }}=\frac{Q+W}{W}
$$

mentre in fase di raffrescamento il calcolo del COP avviene mediante l'equazione [2]:

$$
C O P_{\text {cooling }}=\frac{Q}{W}
$$

L'indice di efficienza energetica stagionale (SEER, Seasonal Energy Efficiency Ratio) rappresenta un altro parametro frequentemente utilizzato in fisica tecnica; il SEER definisce il rapporto tra la capacità di raffreddamento netta ed il lavoro applicato nel corso della medesimo periodo di osservazione (Egg, 2011).

Tra i molti vantaggi legati al ricorso alle pompe di calore si ricordano le contenute spese di esercizio dell'impianto, i minori costi energetici per la climatizzazione, l'assenza di combustione negli ambienti, i contenuti volumi d'ingombro e - non da ultimo - le ridotte emissioni di $\mathrm{CO}_{2}$ legate a questa tecnologia!

\section{BIBLIOGRAFIA}

Banks D (2008) An Introduction to Thermogeology: Ground Source Heating and Cooling, 1st edn Wiley-Blackwell

Cultrera M. (2013) GEOTERMIA-Energia geotermica o geoscambio? Acque Sotterranee 2: 47-48 DOI:

10.7343/AS-031-13-0056

Egg J and Howard B.C. (2011) Geothermal HVAC. Green Heating and Cooling. McGraw-Hill

Freeston D.H. and H. Pan, The application and design of downhole heat exchanger, Geothermics, 14 (1985) 343-351

Huenges E. (2010) - Geothermal Energy Systems. WILEYVCH Verlag GmbH \& Co. KGaA, Weinheim

Huttrer GW (1997) Geothermal heat pumps: An increasingly successful technology. Renewable Energy 10: 481-488 DOI: 10.1016/0960-1481(96)00107-3 